\title{
Transfert de chaleur par convection thermoélectrique dans des cavités rectangulaires horizontales
}

\section{Heat transfer by thermoelectric convection in horizontal rectangular cavities}

\author{
Elhadj BARRY ${ }^{1}$, Changwoo KANG ${ }^{2}$, Harunori YOSHIKAWA ${ }^{3}$, Innocent MUTABAZI ${ }^{1}$ \\ ${ }^{1}$ Normandie Université, UNIHAVRE, LOMC UMR 6294 CNRS, Le Havre, France, elhadj-boubacar.barry@univ-lehavre.fr, \\ innocent.mutabazi@univ-lehavre.fr \\ ${ }^{2}$ Department of Mechanical Engineering, Jeonbuk National University, Deokjin-gu, Jeonju-si, Jeollabuk-do, 54896, \\ Republic of Korea, changwoo.kang@jbnu.ac.kr \\ ${ }^{3}$ Intitut de Physique de Nice, UMR 7010 - Université Côte d'Azur, 1361 Route des Lucioles, 06560 Valbonne, France \\ harunori.yoshikawa@unice.fr
}

RÉSUMÉ. Cette étude concerne la convection naturelle dans un fluide diélectrique dans une cavité rectangulaire soumise à un gradient de température et à un champ électrique alternatif de haute fréquence. Le couplage thermoélectrique induit une force diélectrophorétique qui a des effets similaires à ceux de la poussée d'Archimède dans la génération de la convection thermique. L'analyse de stabilité linéaire a permis de déterminer les modes critiques. Au-delà du seuil, nous avons réalisé des simulations numériques pour caractériser l'écoulement du fluide ainsi que l'efficacité du champ électrique sur le transfert thermique. Nous avons introduit un nouveau paramètre de contrôle, englobant la poussée d'Archimède et la poussée électrique, pour généraliser le problème de convection thermoélectrique.

ABSTRACT. This study concerns the natural convection in a dielectric fluid confined in a rectangular cavity subjected to a temperature gradient and a high frequency alternating electric field. The thermoelectric coupling induces dielectrophoretic force with effects similar to those of Archimedean buoyancy in generating thermal convection. We conducted a linear stability analysis to find the critical modes. Beyond the threshold, numerical simulation was performed to analyze the flow when increasing the electric voltage and we highlighted the heat transfer enhancement by the electric buoyancy. We have introduced a new control parameter, encompassing the Archimedean buoyancy and the electric buoyancy, to generalize the thermoelectric convection problem.

MOTS-CLÉS. Cavité rectangulaire, Fluide diélectrique, Force diélectrophorétique, Poussée électrique, Convection thermoélectrique.

KEYWORDS. Rectangular cavity, Dielectric fluid, Dielectrophoretic force, Electric buoyancy, Thermoelectric convection.

\section{Nomenclature}




\begin{tabular}{|c|c|c|c|}
\hline Symbole & Signification, unités & Symbole & Signification, unités \\
\hline$d$ & épaisseur de la couche de fluide, $\mathrm{m}$ & $P r$ & nombre de Prandtl \\
\hline$l_{x}$ & longueur de la cavité, $\mathrm{m}$ & $N u$ & nombre de Nusselt \\
\hline$l_{y}$ & largeur de la cavité, $\mathrm{m}$ & $R a$ & nombre de Rayleigh thermique \\
\hline$u$ & vitesse horizontale suivant $x, \mathrm{~ms}^{-1}$ & $U$ & amplitude de $u$ perturbée \\
\hline$v$ & vitesse horizontale suivant $y, \mathrm{~ms}^{-1}$ & $V$ & amplitude de $v$ perturbée \\
\hline$w$ & vitesse verticale suivant $z, \mathrm{~ms}^{-1}$ & $W$ & amplitude de $w$ perturbée \\
\hline$D$ & opérateur différentiel par rapport à $z$ & $R a^{\prime}$ & nombre Rayleigh modifié \\
\hline$E$ & champ électrique, $\mathrm{V} / \mathrm{m}^{\prime}$ & & Symboles grecs \\
\hline$\Delta T$ & différence de température, $\mathrm{K}$ & $\nu$ & viscosité cinématique, $\mathrm{m}^{2} / \mathrm{s}$ \\
\hline$f_{D E P}$ & force diélectrophorétique, $\mathrm{kgm}^{-2} \mathrm{~s}^{-2}$ & $\kappa$ & diffusivité thermique, $\mathrm{m}^{2} / \mathrm{s}$ \\
\hline$P_{E}$ & poussée électrique, $\mathrm{kgm}^{-2} \mathrm{~s}^{-2}$ & $\epsilon$ & permittivité électrique, $\mathrm{F} / \mathrm{m}$ \\
\hline$g$ & gravité terrestre, $\mathrm{ms}^{-2}$ & $\sigma$ & taux de croissance temporel \\
\hline$g_{e}$ & gravité électrique, $\mathrm{ms}^{-2}$ & $\alpha$ & coéfficient d'expansion thermique, $\mathrm{K}^{-1}$ \\
\hline$T_{0}$ & température de référence, $\mathrm{K}$ & $\phi$ & potentiel électrique, $\mathrm{V}$ \\
\hline$k_{x}$ & nombre d'onde suivant $x$ & $\theta$ & déviation de la température, $\mathrm{K}$ \\
\hline$k_{y}$ & nombre d'onde suivant $y$ & $\pi$ & pression totale, $\mathrm{kgm}^{-1} \mathrm{~s}^{-2}$ \\
\hline$s$ & taux de croissance complexe & $\beta$ & gradient de température vertical, $\mathrm{Km}^{-1}$ \\
\hline$q$ & module du nombre d'onde & $\gamma_{e}$ & coefficient de couplage thermoélectrique \\
\hline$L$ & nombre de Rayleigh électrique & $\omega$ & fréquence de la perturbation, $\mathrm{Hz}$ \\
\hline
\end{tabular}

\section{Introduction}

Le développement des puces électroniques comportant des composants à base de polymères a permis de mettre au point des méthodes de fabrication de systèmes thermiques, fluidiques ou électromécaniques de tailles millimétriques et submillimétriques [1]. Cependant, la maîtrise des échanges thermiques dans ces appareils miniaturisés reste aujourd'hui un défi majeur de la technologie. Beaucoup de systèmes de refroidissement d'équipements industriels de nos jours utilisent le phénomène de convection naturelle pour réguler le transfert de chaleur $[2,3]$. Ce mécanisme est causé par la poussée d'Archimède et se déclenche spontanément lorsque la différence de température $\Delta T$ atteint une valeur critique $\Delta T_{c}$. Lorsque le fluide est soumis à de faibles écarts de température et dans des conditions de micro-pesanteur, l'utilisation d'un champ électrique est une alternative permettant de créer la convection thermique. Cette méthode trouve son application dans l'industrie aéronautique, aérospatiale et dans la microfluidique. 
Plusieurs études ont étés menées sur les effets de la force diélectrophorétique sur une couche de fluide diélectrique horizontale $[4,5,6]$. Il a été montré que la gravité électrique s'oriente toujours de la plaque froide vers la plaque chaude. Quand la paroi inférieure est plus chaude que la paroi supérieure, la gravité électrique s'ajoute donc à la gravité terrestre; tandis que lorsque le système est chauffé par le haut, les deux gravités sont opposées. Dans ces deux cas de stratification du fluide (stable ou instable), il est possible de déstabiliser l'état conductif de base du système par le champ électrique et de créer de la convection en présence ou en absence de la gravité terrestre; la valeur du champ électrique critique correspondant au déclenchement de la convection dépend du nombre de Rayleigh gravitationnel $R a$ [7, 8, 9]. Dans ces travaux, les auteurs ont montré que les valeurs des paramètres critiques ne dépendent pas de la nature du fluide. Toutefois, les études de stabilité linéaire ont été réalisées uniquement pour quelques valeurs du nombre de Rayleigh thermique $R a$ en considérant des perturbations bidimensionnelles. Nos travaux proposent de compléter ces analyses de stabilité linéaire en introduisant des perturbations tridimensionnelles pour trouver le seuil des instabilités thermoélectriques. Les résultats seront comparés à ceux obtenus dans la littérature. Nous allons ensuite faire une étude non linéaire au-delà du seuil pour caractériser les différentes structures générées par le couplage thermoélectrique et quantifier le transfert de chaleur.

Dans cet article, nous présentons la formulation mathématique du problème dans la section $(\$ 2)$; 1'analyse de stabilité linéaire et les résultats sont présentés dans la section (§3). Ensuite, les résultats des simulations numériques seront présentés dans la section $(\S 4)$ et nous terminerons par une conclusion en section $(\$ 5)$.

\section{Formulation du problème}

\subsection{Force diélectrophorétique}

Lorsqu'une couche de fluide diélectrique de masse volumique $\rho$ et de permittivité $\epsilon$ est soumise à une différence de température constante $\Delta T$, l'application d'un champ électrique alternatif de haute fréquence $\vec{E}$ induit une force volumique appelée force diélectrophorétique (DEP) [10] :

$$
\vec{f}_{D E P}=-\frac{1}{2} \vec{E}^{2} \vec{\nabla} \epsilon
$$

En considérant que la masse volumique et la permittivité varient linéairement en fonction de la température selon l'approximation de Boussinesq, la décomposition de la force DEP contient une partie non conservative appelée poussée électrique $\vec{P}_{E}$ avec une gravité électrique effective $\vec{g}_{e}$ :

$$
\vec{P}_{E}=-\alpha \rho \theta \vec{g}_{e} \quad \text { avec } \quad \vec{g}_{e}=\frac{e}{\alpha \rho} \vec{\nabla}\left(\frac{\epsilon_{1} \vec{E}^{2}}{2}\right)
$$

où $\alpha$ est le coefficient d'expansion thermique, $\theta=T-T_{0}$ la déviation de la température à la température de référence $T_{0}$, e le coefficient de variation de la permittivité, et $\epsilon_{1}$ la permittivité du fluide diélectrique prise à la température $T_{0}=\frac{T_{1}+T_{2}}{2}$. L'expression de la gravité électrique (Eq.2) montre que son intensité est proportionnelle à la densité de l'énergie du champ électrique emmagasinée dans la couche du fluide. 


\subsection{Configuration géométrique et équations générales}

Considérons un fluide diélectrique de viscosité $\nu$ et de diffusivité thermique $\kappa$ placé entre deux plaques horizontales; ces plaques sont maintenues à des températures différentes et servent d'électrodes pour l'application du champ électrique. Le fluide a une épaisseur de $d$; la largeur et la longueur du système sont considérées infiniment grandes par rapport à la distance $d$.

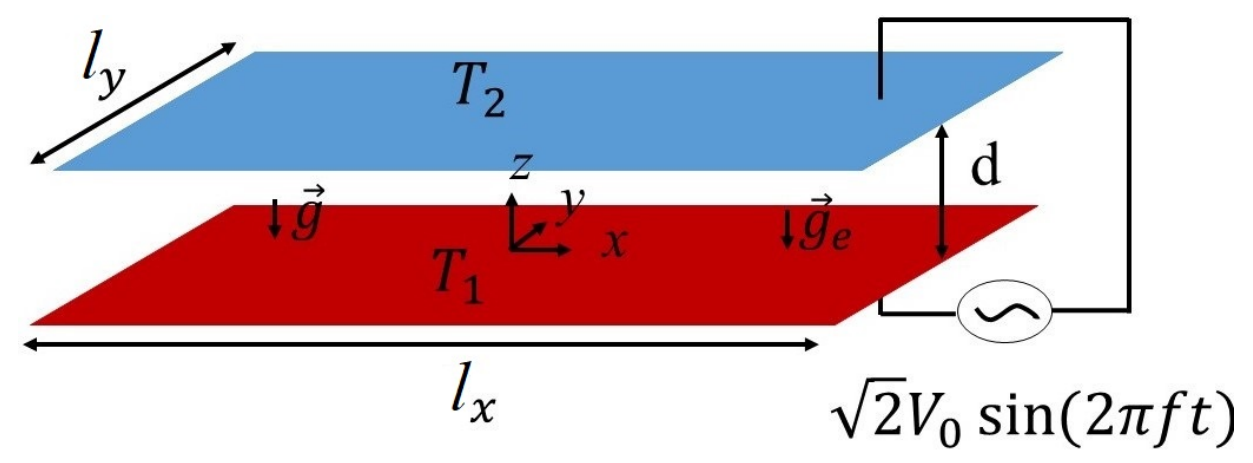

FIGURE 1.: Couche horizontale de fluide diélectrique soumise à une différence de température et à un champ électrique alternatif de haute fréquence.

La nature diffusive du fluide est décrite par le nombre de Prandtl $\operatorname{Pr}=\nu / \kappa$, l'intensité de la différence de température par le nombre de Rayleigh $R a=\alpha \Delta T g d^{3} / \nu \kappa$, et l'intensité du champ électrique appliqué par le nombre de Rayleigh électrique $L=\alpha \Delta T \bar{g}_{e} d^{3} / \nu \kappa$. L'écoulement dans la cavité est régi par l'équation de continuité, l'équation de Navier-Stokes, l'équation de l'énergie, et l'équation de Gauss qui sont données, dans l'approximation de Boussinesq, par :

$$
\left.\begin{array}{c}
\vec{\nabla} \cdot \vec{u}=0, \\
\frac{\partial \vec{u}}{\partial t}+(\vec{u} \cdot \vec{\nabla}) \vec{u}=-\vec{\nabla} \pi+\Delta \vec{u}-\alpha \theta\left(\vec{g}+\overrightarrow{g_{e}}\right), \\
\frac{\partial \theta}{\partial t}+(\vec{u} \cdot \vec{\nabla}) \theta=\kappa \Delta \theta, \\
\vec{\nabla} \cdot[\epsilon(\theta) \vec{\nabla} \phi]=0, \quad \text { avec } \quad \vec{E}=-\vec{\nabla} \phi,
\end{array}\right\}
$$

où $\vec{u}(x, y, z)=(u, v, w)$ est le champ de vitesse, $\phi$ le potentiel électrique, et $\pi=\frac{p}{\rho_{0}}+g z+\frac{e \epsilon_{1} \theta \vec{E}^{2}}{2 \rho_{0}}-$ $\frac{1}{2}\left(\frac{\partial \epsilon}{\partial \rho}\right)_{T} \vec{E}^{2}$ la fonction de Bernoulli qui représente la charge hydraulique généralisée. La vitesse, la température et le potentiel électrique satisfont les conditions aux limites suivantes :

$$
\begin{array}{llll}
\text { à } & z=-d / 2, \quad \vec{u}=0, \quad \theta=\Delta T / 2, & \phi=\phi_{0} ; \\
\text { à } & z=d / 2, \quad \vec{u}=0, \quad \theta=-\Delta T / 2, & \phi=0 .
\end{array}
$$

Pour des faibles valeurs de l'écart de température et du potentiel électrique, le système admet un régime permanent de conduction pure $(\vec{u}=0)$. La température et le champ électrique de l'état de base dépendent uniquement de la direction verticale $z$ :

$$
\theta_{b}=-\beta z, \quad E_{b}=\frac{E_{1}}{\left(1+\gamma_{e}\left(\frac{z}{d}\right)\right)}, \quad \text { avec } \quad \beta=\frac{\Delta T}{d} \quad \text { et } \quad E_{1}=-\frac{\phi_{0}}{d} \frac{\gamma_{e}}{\log \left(\frac{2-\gamma_{e}}{2+\gamma_{e}}\right)} .
$$


Le paramètre $\gamma_{e}=e \Delta T$ représente le coefficient de couplage thermoélectrique. L'expression de la gravité électrique de l'état de base est donnée par $[9,11,12]$ :

$$
g_{e b}=\bar{g}_{e}\left[1+\gamma_{e}\left(\frac{z}{d}\right)\right]^{-3}, \quad \text { avec } \quad \bar{g}_{e}=-\frac{\epsilon_{1} e \phi_{0}^{2}}{\rho_{0} \alpha}\left(\frac{\gamma_{e}}{d}\right)^{3}\left[\log \left(\frac{2-\gamma_{e}}{2+\gamma_{e}}\right)\right] .
$$

La quantité $\bar{g}_{e}$ correspond à la valeur de la gravité électrique à $z=0$.

\section{Analyse de stabilité linéaire}

Les équations (3) de la section (\$2) sont adimensionnées en utilisant les échelles suivantes : $d$ pour la longueur, le temps visqueux $\tau_{\nu}=d^{2} / \nu$ pour le temps, la vitesse visqueuse $\nu / d$ pour la vitesse, $\Delta T$ pour la température, $\rho(\nu / d)^{2}$ pour la pression et la quantité $\phi_{0} / d$ pour le champ électrique. On ajoute des perturbations infinitésimales de vitesse, de température, de pression et de potentiel électrique $\left(u^{\prime}, v^{\prime}, w^{\prime}, \theta^{\prime}, \pi^{\prime}, \phi^{\prime}\right)$ à l'état de base. Nous linéarisons ensuite les équations obtenues autour de l'état de base et on développe les perturbations en modes normaux de la forme $e^{s t+i\left(k_{x} x+k_{y} y\right)}$; où $s=\sigma+i \omega, \sigma$ est le taux de croissance temporel de la perturbation et $\omega$ sa fréquence, $k_{x}$ et $k_{y}$ sont les nombres d'ondes de la perturbation dans les directions $x$ et $y$ respectivement. Le problème de stabilité linéaire est donc formulé comme suit :

$$
\begin{aligned}
0 & =D W+i\left(k_{x} U+k_{y} V\right), \\
s U & =-i k_{x} \Pi+\left(D^{2}-q^{2}\right) U-\frac{L}{P r} \tilde{\theta}_{b} G_{e x}, \\
s V & =-i k_{y} \Pi+\left(D^{2}-q^{2}\right) V-\frac{L}{P r} \tilde{\theta}_{b} G_{e y}, \\
s W & =-D \Pi+\left(D^{2}-q^{2}\right) W+\left(\frac{R a-L \tilde{g}_{e b}}{P r}\right) \Theta-\frac{L}{P r} \tilde{\theta}_{b} G_{e z}, \\
s \Theta & =W+\frac{1}{P r}\left(D^{2}-q^{2}\right) \Theta, \\
0 & =\left[\left(1+\gamma_{e} \tilde{z}\right)\left(D^{2}-q^{2}\right)+\gamma_{e} D\right] \Phi-\gamma_{e}\left(D^{2} \tilde{\phi}_{b}+D \tilde{\phi}_{b} D\right) \Theta,
\end{aligned}
$$

où $q=\left(k_{x}^{2}+k_{y}^{2}\right)^{1 / 2}$ le module du nombre d'onde, l'opérateur différentiel $D \equiv d / d \tilde{z}$ et $[U, V, W, \Theta, \Pi, \Phi]$ sont les amplitudes du mode normal de la perturbation. Nous devons noter que toutes les grandeurs physiques sont additionnées, suite à l'introduction des grandeurs caractéristiques. L'écoulement du fluide diélectrique est stabilisé par la dissipation visqueuse et la diffusion thermique tandis que la gravité terrestre et la poussée électrique représentent les effets déstabilisants du système. Pour mettre en évidence les effets de la poussée électrique, le nombre de Rayleigh thermique $R a$ est choisi tel que sa valeur soit inférieure à la valeur seuil $R a_{c}=1708$ de la convection de Rayleigh-Bénard entre deux plaques parallèles. Les conditions aux limites pour les perturbations sont données par :

$$
U=V=W=\Theta=\Pi=\Phi=0 \quad \text { à } \quad \tilde{z}= \pm 1 / 2 .
$$

Le système d'équations linéarisées est résolu avec un code basé sur une méthode spectrale. Les fonctions inconnues $[U, V, W, \Theta, \Pi, \Phi]$ sont développées en séries de polynômes de Chebyshev et les solutions sont cherchées aux points de collocations de Chebychev-Gauss-Lobatto. Le nombre de polynômes 
de Chebychev a été fixé à 32 pour assurer la convergence des résultats. Le problème réultant est ainsi résolu par la méthode de décomposition QZ [9].

Pour des valeurs fixes du $\operatorname{Pr}$, nous avons déterminé les courbes marginales de stabilité $(\sigma=0)$ pour différentes valeurs des paramètres de contrôle $(R a, L)$. Pour cela, le nombre de Rayleigh $R a$ est fixé et nous faisons varier le nombre de Rayleigh électrique $L$ pour des valeurs du nombre d'onde $q$ comprises dans l'intervalle de $[0 ; 5]$. Nous présentons quelques résultats des conditions marginales sur la figure 2.

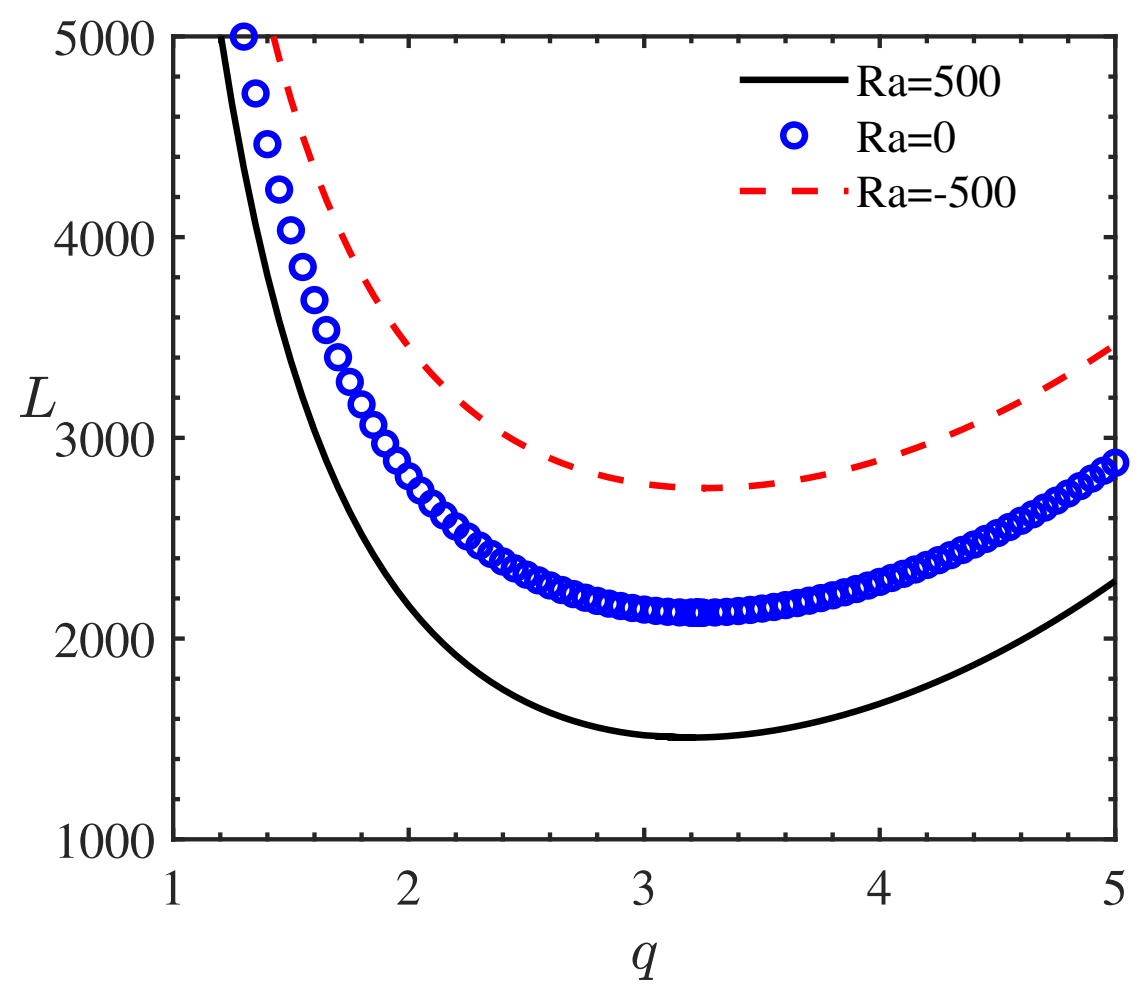

Figure 2.: Courbes de stabilité marginale pour $\operatorname{Pr}=1$ et différentes valeurs de $R a$ : la courbe noire correspond à $R a=500$, les cercles bleus à $R a=0$ et les pointillés rouges à $R a=-500$.

Le minimum des courbes marginales de stabilité donnent les valeurs des paramètres critiques $\left(L_{c}, q_{c}, \omega_{c}\right)$. Les résultats obtenus sont présentés dans le tableau 3.1. Les modes critiques sont des modes stationnaires $\left(\omega_{c}=0\right)$. Lorsque le fluide est instable par rapport à la poussée d'Archimède $(R a>0)$, l'état de base du système est déstabilisé pour de faibles valeurs du nombre de Rayleigh électrique $L$; et quand le fluide est stable par rapport aux effets de la gravité terrestre $(R a<0)$, il faut un champ électrique suffisamment fort pour induire des instabilités. Le seuil des instabilités diélectrophorétiques dépend du nombre de Rayleigh gravitationnel $R a$ et les valeurs des paramètres critiques sont indépendantes du nombre de Prandtl $(P r)$.

Les résultats obtenus ici avec les perturbations tridimensionnelles sont en parfait accord avec les résultats donnés par Roberts [7], et Stiles [8] avec des perturbations bidimensionnelles. 


\begin{tabular}{c|ccccc}
\hline \hline$R a$ & -1000 & -500 & 0 & 500 & 1000 \\
\hline$L_{c}$ & 3370.07 & 2749.89 & 2128.70 & 1506.57 & 883.52 \\
\hline$q_{c}$ & 3.29 & 3.26 & 3.23 & 3.19 & 3.16 \\
\hline \hline
\end{tabular}

TABleau 3.1.: Paramètres critiques $\left(L_{c}, q_{c}\right)$ pour $\operatorname{Pr}=1$ et différentes valeurs de Ra

\section{Simulation numérique directe}

Après avoir déterminé les paramètres critiques par une analyse de stabilité linéaire, nous avons fait une étude non linéaire des instabilités thermoélectriques. Les simulations numériques ont été réalisées à l'aide d'un code basé sur la méthode des volumes finis [12]. Une méthode hybride a été utilisée pour la discrétisation temporelle : le schéma de Cranck-Nicolson pour les termes non linéaires et diffusifs tandis que nous avons employé le schéma de Runge-Kutta d'ordre 3 pour les autres termes. Nous avons utilisé les conditions aux limites de Dirichlet pour la vitesse, la température et le potentiel électrique sur les électrodes; et les conditions aux limites périodiques dans les directions horizontales $x$ et $y: \vec{u}(x, y, z)=$ $\vec{u}\left(x \pm l_{x} / 2, y \pm l_{y} / 2, z\right), \theta(x, y, z)=\theta\left(x \pm l_{x} / 2, y \pm l_{y} / 2, z\right)$ et $\phi(x, y, z)=\phi\left(x \pm l_{x} / 2, y \pm l_{y} / 2, z\right)$ où $l_{x}=l_{y}=10$. Un maillage régulier est adopté pour les directions horizontales avec 256 points suivant $x$ et $y$ tandis que le maillage est plus raffiné près des parois avec $\Delta z=0.005$ suivant la direction verticale $z$. La valeur du coefficient de couplage thermoélectrique est fixée à $\gamma_{e}=0.01$ et $\operatorname{Pr}=1$ pour les simulations.

Les résultats montrent que près du seuil, les instabilités se manifestent sous forme de rouleaux de convection droits stationnaires d'axe incliné dans le plan $(x, y)$. La figure 3 montre l'évolution des structures présentes dans l'écoulement pour différentes valeurs de $L$. Les rouleaux, initialement droits, deviennent modulés avec des défauts stationnaires, ensuite oscillants $(L=7000 ; 8000)$ avec des défauts de type dislocations, similaires à ceux obtenus dans la convection de Rayleigh-Bénard pour de grandes valeurs du $R a$ [13].

Les figures 4 et 5 illustrent des cellules de convection de la taille de l'épaisseur du fluide pour certaines valeurs de $L$ dans des conditions de microgravité. Certains de ces motifs ont été observés expérimentalement dans des huiles de silicone lors d'une campagne de vols paraboliques. Les résultats ont été présentés par A. Meyer dans les actes du Groupement De Recherche Micro-pesanteur Fondamentale et Appliquée (GDR-MFA) en 2020 [14].

Pour caractériser le transfert thermique par convection, nous avons calculé le nombre de Nusselt pour toutes les valeurs de $L$. Par définition, ce nombre sans dimension représente le rapport entre le flux de chaleur total (conduction + convection) et le flux de chaleur par conduction :

$$
N u=\frac{1}{l_{x} l_{y}} \int_{-\frac{l_{y}}{2}}^{\frac{l_{y}}{2}} \int_{-\frac{l_{x}}{2}}^{\frac{l_{x}}{2}}\left(\operatorname{Pr} w \theta-\frac{\partial \theta}{\partial z}\right) d x d y .
$$



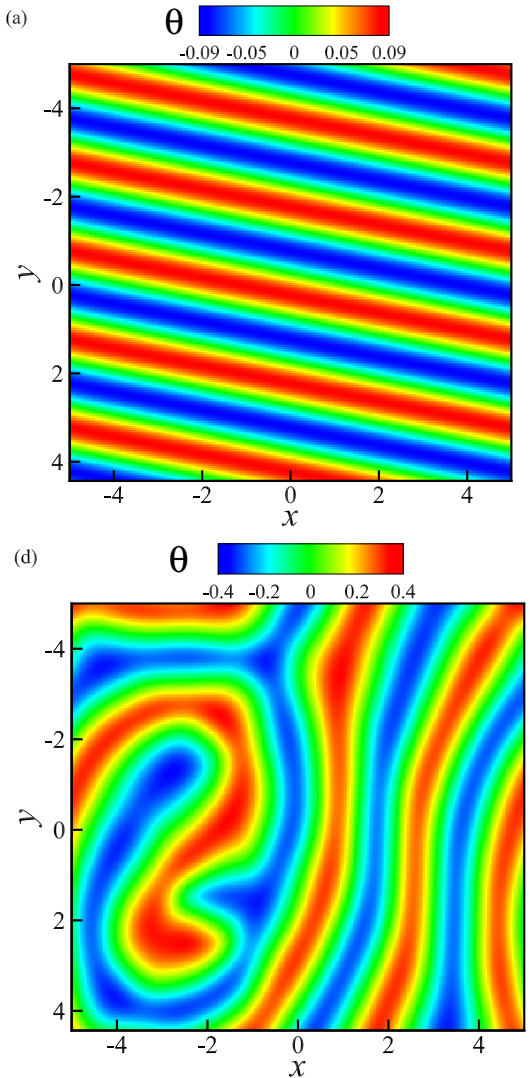
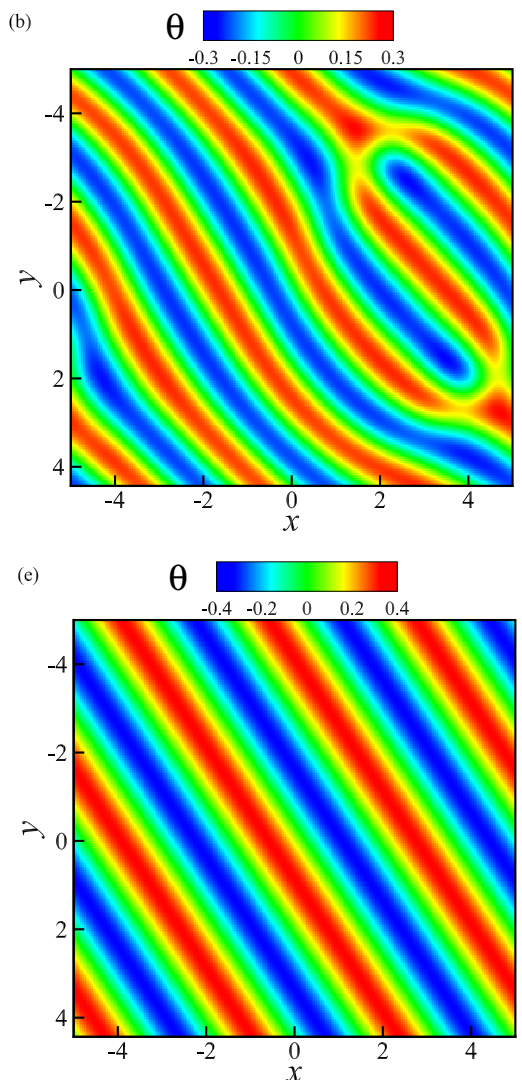
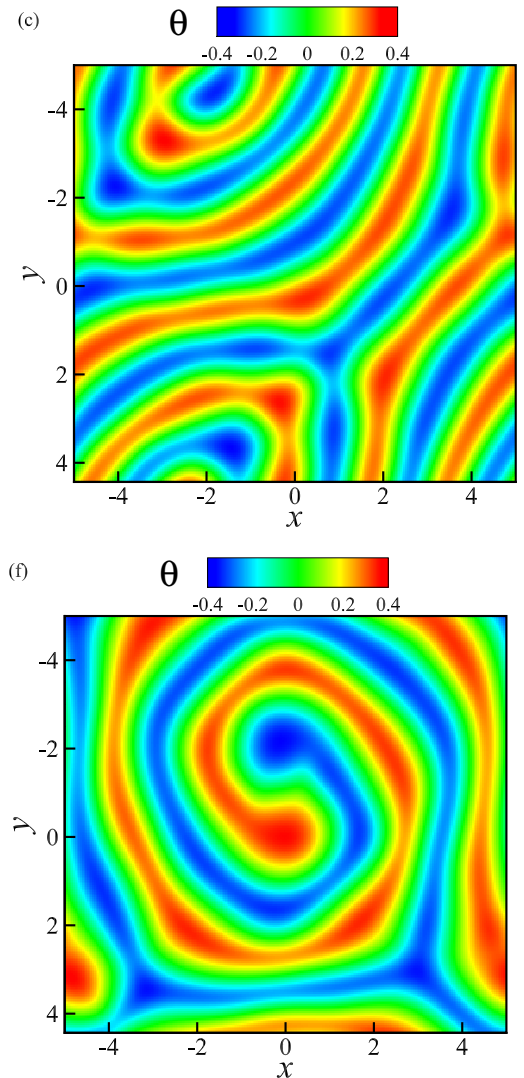

Figure 3.: Champ de température instantané dans le plan $z=0$ pour $\operatorname{Pr}=1, R a=-500$ et différentes valeurs de $L$ : (a) $L=2850$, (b) $L=4000$, (c) $L=5000$, (d) $L=7000$, (e) $L=7500$, et (f) $L=8000$.

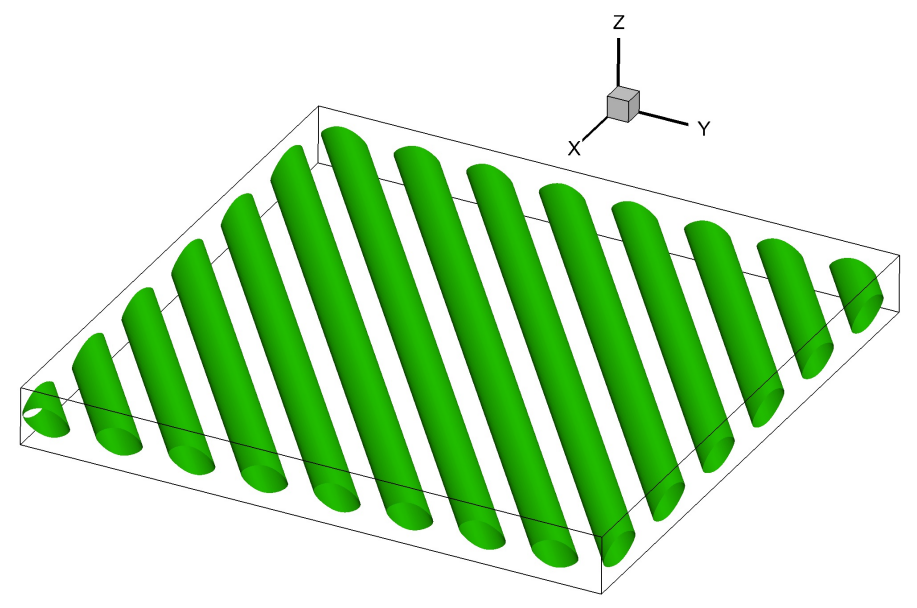

FIgURE 4.: Structures des iso-surfaces de $Q=40$ pour $R a=0$ et $L=2400$.

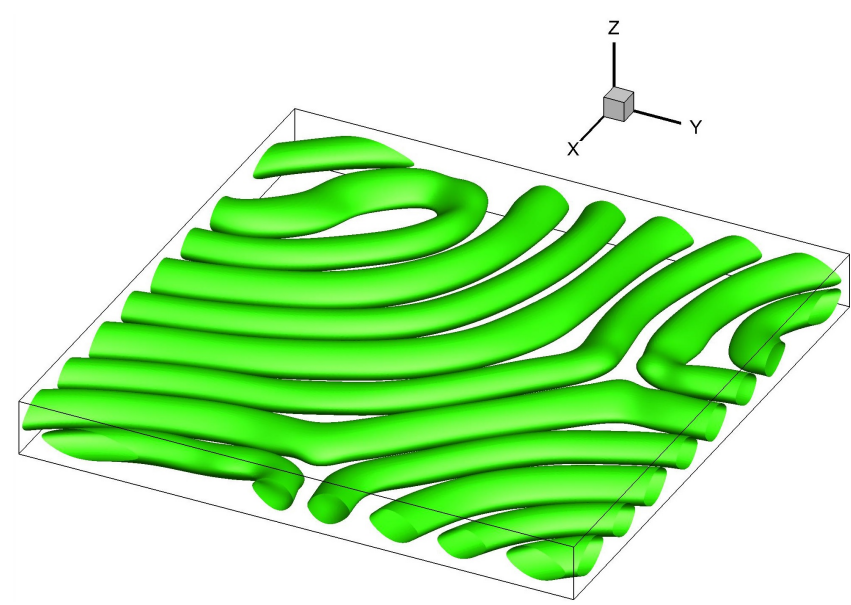

FIGURE 5.: Structures des iso-surfaces de $Q=$ 200 pour $R a=0$ et $L=5000$. 
Lorsque le fluide est au repos $\left(L<L_{c}\right)$, le transfert de chaleur se fait uniquement par conduction, par conséquent le nombre de Nusselt $N u=1$. Pour quantifier l'apport des instabilités thermoélectriques sur le transfert thermique, nous avons tracé sur la figure 6, l'évolution du $N u-1$ en fonction du nombre de Rayleigh électrique $L$ pour différentes valeurs du Rayleigh thermique $R a$. Quelque soit la valeur du nombre de Rayleigh $R a$, on constate un accroissement du nombre de Nusselt $N u$ en fonction du nombre de Rayleigh électrique lorsque $L>L_{c}$. Nous remarquons que les courbes du $N u-1$ sont plus basses que celle de $R a=0$ lorsque $R a$ est négatif. Cela est dû à l'effet stabilisant de la poussée d'Archimède sur l'écoulement du fluide diélectrique dans cette configuration.

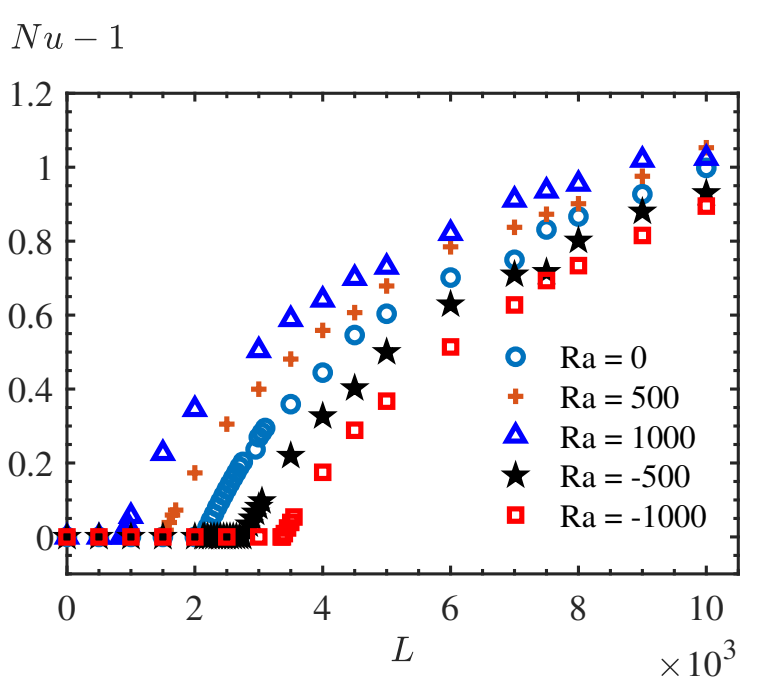

Figure 6.: Variation du nombre de Nusselt $N u$ en fonction du nombre de Rayleigh électrique $L$ pour $\operatorname{Pr}=1$ et quelques valeurs du nombre de Rayleigh thermique Ra.

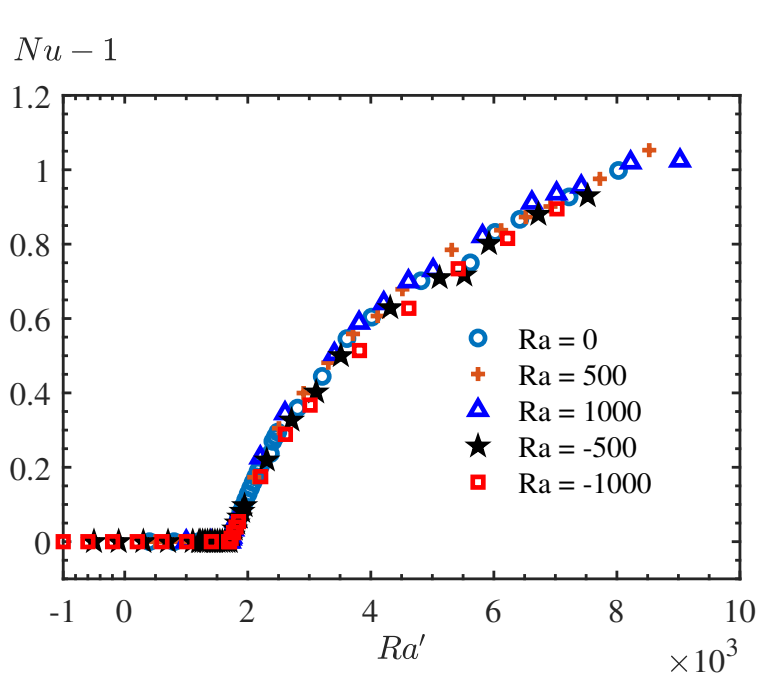

Figure 7.: Variation du nombre de Nusselt $N u$ en fonction du nombre de Rayleigh modifié $R a^{\prime}$ pour $\operatorname{Pr}=1$, Ra donné et différentes valeurs croissantes de $L$

Pour normaliser les courbes du Nusselt $N u$, nous avons introduit le nombre de Rayleigh modifié $R a^{\prime}$. Ce nombre, englobant les deux paramètres de contrôle $R a$ et $L$, a été défini en tenant compte des seuils des instabilités du problème de Rayleigh-Bénard classique $\left(L=0, R a_{c}=1708\right)$ et de la convection diélectrophorétique dans des condition de microgravité $\left(R a=0, L_{c}=2129\right)$ :

$$
R a^{\prime}=R a+\left(R a_{c} / L_{c}\right) \cdot L .
$$

Pour tout couple $(R a, L)$, le comportement global du $N u-1$ peut donc être tracé en fonction du nombre de Rayleigh modifié $R a$ (figure 7). On observe que toutes les courbes sont presque superposées. De plus, le seuil de la convection thermoélectrique $\left(L=L_{c}\right)$ correspond à des valeurs de $R a^{\prime}$ comprises entre 1704 (pour $R a=-1000$ ) et 1709 (pour $R a=1000$ ) quelque soit la valeur du nombre de Rayleigh gravitationnel $R a$. Ceci ramène le problème généralisé de la convection thermique induite par la poussée électrique à un problème classique de Rayleigh-Bénard avec $R a^{\prime}$ comme paramètre de contrôle. 


\section{Conclusion}

Nous avons réalisé une étude de la convection thermique induite par la poussée électrique dans une couche horizontale de fluide diélectrique soumise à un gradient vertical de température pour différentes valeurs du champ électrique. L'analyse de stabilité linéaire avec des perturbations 3D a permis de déterminer les valeurs critiques marquant le début de la convection thermoélectrique. Les modes critiques correspondants se manifestent par des rouleaux stationnaires indépendants de la nature du fluide. Les simulations numériques ont montré qu'au-delà du seuil critique, des rouleaux convectifs oscillants de type Rayleigh-Bénard ainsi que des dislocations apparaissent dans l'écoulement. Le développement de la convection s'accompagne d'un accroissement de la valeur du nombre de Nusselt. Cela prouve que la force DEP est un moyen alternatif permettant de créer des mouvements convectifs dans un fluide soumis à l'action du champ gravitationnel ou dans des conditions de microgravité. Cette méthode pourrait être utilisée dans des systèmes miniaturisés où il est possible d'appliquer des champs électriques intenses. L'implémentation de cette technique aux systèmes de refroidissement est d'un grand intérêt pour les industries aéronautique et aérospatiale où la réduction du poids ainsi que la taille des composants, tout en gardant les mêmes fonctionnalités, est un facteur primordial.

\section{Remerciements}

Ces travaux de thèse sont co-financés par le Centre National d'Études Spatiales (CNES) dans le cadre du projet INTEHLDI et la Région de Normandie.

\section{Bibliographie}

[1]. KiRBY B. J., Micro- and Nanoscale Fluid Mechanics. Cornell University - New York : Cambridge University Press, 2010.

[2]. BaÏri A., Zarco-Permia E., Garcia de MariaJ.M., «A review on natural convection in enclosures for engineering », Int. J. Fond. Therm., 63 (304-322), 2014.

[3]. Miroshnichenko I.V., Sheremet M.A., «Turbulent natural convection heat transfer in rectangular enclosures using experimental and numerical approaches : A review », Renew. Sustain. Energy Rev., 32 (40-59), 2018.

[4]. Gross M.J. and PorTer J.E., «Electrically induced convection in dielectric liquids », Nature, 2012 (1343-1345), 1966.

[5]. Turnbull R.J., «Effect of dielectrophoretic forces on the Bénard instability », Phys. Fluids, 12 (1809-1815), 1969.

[6]. Mutabazi I., Yoshikawa H., Tadie Fogaing M., Travnikov V., Crumeyrolle O., Futterer B. and EGBERS C., «Thermo-electro-hydrodynamic convection under microgravity : a review », Fluid Dynamics Research, 48 (061413), 2016.

[7]. Roberts P.H., «Electrohydrodynamic convection », Q. J. Mech. Appl. Math., 22 (211-220), 1969.

[8]. STILES P.J., «Electro-thermal convection in dielectric liquids », Chem. Phys. Lett., 179 (311-315), 1991.

[9]. Yoshikawa H.N., Tadie Fogaing M., Crumeyrolle O. and Mutabazi I., «Dielectrophoretic Rayleigh-Bénard convection under microgravity conditions », Phys. Rev. E., 87 (043003), 2013.

[10]. LANDAu L. D., and Lifshitz E. M., Electrodynamics of Continuous Media, Landau and Lifshitz Course of Theoretical Physics. Burlington-Massachusetts - Elsevier Butterworth-Heinemann, 1984.

[11]. Barry E. B., Yoshikawa H.N., Tadie Fogaing M., Kang C. and Mutabazi I., « Critical Modes of Thermoelectric Convection Instabilities in a Vertical Slot », Microgravity Science and Technology, 33 (1875-0494), 2021.

[12]. KANG C. and Mutabazi I., "Columnar vortices induced by dielectrophoretic force in a stationary cylindrical annulus filled with a dielectric liquid », Journal of Fluid Mechanics, 908 (A26), 2021. 
[13]. Manneville P., Dissipative Structures and weak Turbulence. Academic Press, 1990.

[14]. Meyer A., Meier M., Barry E. B., Crumeyrolle O., Mutabazi I., Egbers C., «Etude expérimentale de la convection thermo-électro-hydrodynamique dans une cavité rectangulaire en vol parabolique », Micropesanteur Fondamentale et Appliquée, 2020. http ://gdr-mfa.fr/index.php/colloques. 